\title{
La mejora del aprendizaje y el desarrollo de compe- tencias en estudiantes universitarios a través de la colaboración
}

\author{
Ana María Pinto-Llorente, Verónica Basilotta \\ Gómez-Pablos \& Vanessa Izquierdo-Álvarez
}

\begin{abstract}
Resumen:
En la actualidad los estudiantes demandan propuestas de enseñanza más flexibles, activas y participativas, adaptadas a sus necesidades, que fomenten el aprendizaje colaborativo y el desarrollo de diferentes competencias y habilidades. $Y$ es que en el aprendizaje colaborativo los alumnos asumen la responsabilidad de su aprendizaje, reflexionando sobre sus procesos cognitivos y desarrollando habilidades de orden superior. En este contexto, el objetivo de esta investigación es conocer las concepciones de los estudiantes de la Universidad de Salamanca acerca de los beneficios e inconvenientes que posee la metodología de aprendizaje colaborativo en función de su experiencia. Para ello, se ha utilizado un enfoque cuantitativo y cualitativo, a través de un cuestionario que se ha aplicado a una muestra de 137 estudiantes. Los resultados muestran una valoración positiva de esta metodología. Los estudiantes destacan haber aprendido a compartir conocimientos, tener mayor autonomía y control sobre la tarea, ser tolerantes y respetuosos con los demás, solucionar conflictos y resolver problemas. Sin embargo, destacan algunos inconvenientes relacionados con la implicación de ciertos compañeros en el equipo, además del esfuerzo y dedicación adicional que supone a las tareas académicas.
\end{abstract}

Palabras clave:

educación superior; aprendizaje colaborativo; competencias; estudiantes. 


\section{Melhorar a aprendizagem dos alunos e o desenvolvimento de competências através da colaboração na Universidade}

Resumo: actualmente, os estudantes exigem propostas de ensino mais flexíveis, activas e participativas, adaptadas às suas necessidades, que incentivem a aprendizagem colaborativa e o desenvolvimento de diferentes competências e aptidões. Na aprendizagem colaborativa, os alunos assumem a responsabilidade pela sua aprendizagem, reflectindo sobre os seus processos cognitivos e desenvolvendo competências de ordem superior. Neste contexto, o objetivo desta pesquisa é conhecer as concepções dos estudantes da Universidade de Salamanca sobre os benefícios e desvantagens da metodologia de aprendizagem colaborativa baseada na sua experiência. Para tal, foi utilizada uma abordagem quantitativa e qualitativa, através de um questionário aplicado a uma amostra de 137 estudantes. Os resultados mostram uma avaliação positiva desta metodologia. Os alunos enfatizam ter aprendido a compartilhar conhecimento, ter maior autonomia e controle sobre a tarefa, ser tolerante e respeitoso com os outros, resolver conflitos e resolver problemas. No entanto, existem alguns inconvenientes relacionados com o envolvimento de alguns colegas da equipa, para além do esforço extra e dedicação às tarefas académicas.

Palavras-chave: ensino superior; aprendizagem colaborativa; competências; estudantes.

\section{The improvement of learning and the development of University students' competences through collaboration}

Abstract: Nowadays learners demand more flexible, active, and participatory teaching proposals, which are adapted to their needs and encourage collaborative learning and the development of different skills and abilities. Students assume the responsibility of their learning, reflecting about their cognitive processes and developing high-order abilities in the process of collaborative learning. In this context, the objective of the research is to know Salamanca University students' perceptions about the benefits and disadvantages of collaborative learning methodology according to their experience. Based on this purpose, it is employed a quantitative and qualitative approach, administering a questionnaire to a sample of 137 students. The results obtained show a positive assessment of this methodology. The learners highlight that they have learned to share knowledge, to have greater autonomy and control over the task, to be tolerant and respectful to others, and to solve conflicts and problems. However, they also emphasize some disadvantages related to the involvement of some team members and to the effort and additional dedication to the academic tasks that this methodology means.

Keywords: higher education; collaborative learning; competences; students.

\section{Améliorer l'apprentissage et le développement des compétences des étudiants grâce à la collaboration à l'Université}

Résumé: actuellement, les étudiants exigent des propositions d'enseignement plus souples, actives et participatives, adaptées à leurs besoins, qui encouragent l'apprentissage collaboratif et le développement de compétences et d'aptitudes différentes. Dans l'apprentissage collaboratif, les élèves assument la responsabilité de leur apprentissage, réfléchissent sur leurs processus cognitifs et développent des compétences d'ordre supérieur. Dans ce contexte, l'objectif de cette recherche est de connaître les conceptions des étudiants de l'Université de Salamanque sur les avantages et les inconvénients de la méthodologie d'apprentissage collaboratif basée sur leur expérience. Pour ce faire, une approche quantitative et qualitative a été utilisée, au moyen d'un questionnaire appliqué à un échantillon de 137 élèves. Les résultats montrent une évaluation positive de cette méthodologie. Les élèves mettent l'accent sur le fait d'avoir appris à partager leurs connaissances, d'avoir plus d'autonomie et de contrôle sur la tâche, d'être tolérants et respectueux envers les autres, de résoudre les conflits et les problèmes. Cependant, il y a quelques inconvénients liés à l'implication de certains collègues dans l'équipe, en plus de l'effort supplémentaire et du dévouement aux tâches académiques.

Mots-clés: enseignement supérieur; apprentissage collaboratif; compétences; étudiants. 


\section{Introducción}

El aprendizaje colaborativo ha despertado un gran interés en la investigación educativa, lo que ha dado lugar a multitud de estudios e investigaciones, sobre todo, en los últimos años. Estos estudios subrayan las ventajas de la colaboración en los procesos de aprendizaje, de ahí que su integración en la enseñanza universitaria sea una estrategia muy significativa si tenemos en cuenta las exigencias de la sociedad actual, y las capacidades que deben poseer los alumnos del siglo XXI.

Según Yong, Cedeño, Tubay y Cedeño (2018) el aprendizaje colaborativo es una metodología que impulsa la distribución de acciones dentro del aula para enfocarlas en una experiencia social y académica de aprendizaje.

En cierta medida podemos decir que el aprendizaje colaborativo prepara al estudiante para asumir compromisos grupales, proporcionar y pedir ayuda a los compañeros, aprender a aceptar los puntos de vista de los demás, descubrir soluciones que beneficien a todos y aprender a aceptar críticas de otros, exponiendo ideas y planteamientos de forma razonada (Gros, 2011). En este contexto, los estudiantes se convierten en agentes activos en su proceso de aprendizaje y colaboran en la creación de sus propios conocimientos (Blasco, Buil, Hernández y Sese, 2013).

Durante generaciones, los profesores han tratado de buscar el método "óptimo" de enseñanza y se han realizado considerables investigaciones comparando diversos métodos docentes. McKeachie y sus colaboradores, de la Universidad de Michigan, revisaron más de quinientos estudios de investigación relativos a la enseñanza y el aprendizaje en aulas universitarias. Cuando les preguntaron por el método de enseñanza más eficaz respondieron que dependía del objetivo educativo, las características de los estudiantes, el contenido a trabajar y las competencias del docente, aunque la mejor respuesta fue la siguiente: cuando los estudiantes enseñan a otros estudiantes (McKeachie, Pintrich, Lin y Smith, 1986).

El aprendizaje colaborativo ha promovido gran cantidad de investigaciones que centran su atención en valorar los efectos positivos de esta estrategia de enseñanza en diversas variables como el aprendizaje cognitivo, la motivación, la actitud, el desarrollo social y emocional, y el rendimiento académico de los estudiantes (Johnson y Johnson, 2009). En este contexto, destacamos el trabajo de Sobocinski, Malmberg y Jarvela (2017) quienes han identificado que los procesos cognitivos y socioemocionales de los estudiantes son factores que influyen en el aprendizaje colaborativo.

Kolloffel, Eysink y Jong (2011) por su parte destacan la existencia de una larga lista de beneficios ligadas al aprendizaje colaborativo, tales como el desarrollo de la metacognición y un mayor control sobre la tarea. Fomenta que los estudiantes vean las situaciones desde diferentes perspectivas, y crea un ambiente donde los alumnos pueden practicar habilidades sociales y de liderazgo y, por último, proporciona una experiencia de aprendizaje más satisfactoria, lo que reduce significativamente la ansiedad de los alumnos. 
La colaboración promueve la elaboración de nuevos conocimientos en función de la calidad de las interacciones entre los integrantes del equipo, especialmente del proceso de construcción y mantenimiento de la comprensión compartida. También las interacciones afectivas sociales, los sentimientos de cohesión grupal, la orientación en equipo, la confianza mutua y el sentido de comunidad son claves para una colaboración exitosa (Järvelä et al., 2015).

En cuanto a las principales limitaciones para implementar en el aula metodologías de aprendizaje colaborativo, se han señalado algunas cuestiones que tienen implicación en el desarrollo del currículo como el empleo de más tiempo, la diferente implicación de los estudiantes, o las dificultades para evaluar el proceso de aprendizaje, y los resultados obtenidos por cada alumno (García-Valcárcel, Basilotta y López, 2014).

Fransen, Kirschner y Erkens (2011) concluyeron que no todos los equipos colaboran eficazmente. Según estos autores, los alumnos se centran especialmente en el resultado y no en colaborar. La eficacia del aprendizaje colaborativo depende en gran medida de cómo se diseñen y apliquen algunos elementos fundamentales tales como la interdependencia, la responsabilidad individual y la interacción (De Hei, Strijbos, Sjoer y Admiraal, 2015).

Realizada una aproximación teórica inicial del concepto de aprendizaje colaborativo y sus principales virtudes en los procesos de enseñanza, pasamos a describir el desarrollo de una experiencia de aprendizaje colaborativo en la Universidad. La práctica se sustentó como parte de la evaluación continua de una asignatura de primer curso del Grado de Maestro en Educación Primaria, Grado de Maestro en Educación Infantil, y Grado en Pedagogía de la Universidad de Salamanca. Para ello, se solicitó a los participantes la conformación de grupos de un máximo de seis personas, con el objetivo de llevar a cabo el desarrollo de actividades colaborativas fuera y dentro del aula. Los equipos se conformaron de forma libre haciendo especial hincapié en la importancia de la colaboración entre ellos. Los alumnos debían ser responsables de la asignación de roles dentro del mismo y responsabilizarse del trabajo propio y del grupo. Se les informó de los objetivos propuestos para el desarrollo de la experiencia, tales como, el fomento y beneficios del aprendizaje colaborativo, profundización en los contenidos propios de la materia, entre otros. Además, se hizo explícita la importancia de poner en práctica dinámicas organizativas dentro del propio grupo y la colaboración honesta de todos los integrantes.

Una vez descrita la experiencia objeto de estudio, centramos nuestros esfuerzos en indagar qué opinan los estudiantes de la Universidad de Salamanca sobre el aprendizaje colaborativo.

\section{Metodología}

\subsection{Objetivo del estudio}

Este trabajo de investigación tiene por objeto conocer las concepciones de los estudiantes de 1er curso de la Universidad de Salamanca sobre los beneficios e 
inconvenientes que posee la metodología de aprendizaje colaborativo, en función de su experiencia en diversas actividades realizadas durante la asignatura de "Organización del centro escolar" en el Grado de Educación Primaria y Educación Infantil; y la asignatura de "Organización y gestión" en el Grado de Pedagogía. En concreto, las preguntas de investigación mediante las cuales se quiere dar respuesta a este objetivo son: ¿qué aspectos positivos del aprendizaje colaborativo destaca el alumnado?, ¿qué dificultades y limitaciones han encontrado durante la experiencia?

\subsection{Diseño de la investigación}

Este estudio utiliza un enfoque cuantitativo aunque se emplean métodos de carácter cualitativo con el fin de obtener un conocimiento sobre el problema del estudio más amplio y sistemático. Y es que la investigación cualitativa y cuantitativa pueden proporcionar información igualmente valiosa. Si además se integran ambos tipos de datos, cuando estos concurren se produce un refuerzo de la calidad de la investigación (Sánchez, 2015). Flick (2014) resalta la importancia de vincular la investigación cualitativa y la cuantitativa, y señala que pueden beneficiarse mutuamente entre sí. Desde esta postura de la armonía, valoramos igualmente las posibilidades de cada metodología, respetando sus respectivas contribuciones.

\subsection{Población y muestra}

La población objeto de estudio está conformada por 270 estudiantes de la Universidad de Salamanca. De ellos 150 pertenecen a la Facultad de Educación, 79 están matriculados en el 1er año del Grado de Maestro en Educación Infantil y 71 en el Grado de Pedagogía, los otros 120 estudian el Grado de Educación Primaria en la Escuela Universitaria de Educación y Turismo de Ávila.

En base al enfoque de investigación, de los objetivos y del diseño de la misma, se decide emplear un muestreo de tipo no probabilístico, concretamente el muestreo por cuotas. Este muestreo se emplea cuando no se puede disponer de una muestra escogida al azar, pero aún así se quiere una muestra representativa de la población y se fijan unas "cuotas" consistentes en un número de individuos que reúnen determinadas condiciones o variables demográficas en la población. Este método se emplea mucho en las encuestas de opinión (Sabariego, 2004). La muestra se compone de 137 estudiantes, 116 mujeres $(84,7 \%)$ y 21 hombres (15,3\%) con edades comprendidas entre los 18 y los 30 años. En cuanto al Grado en el que se encuentran matriculados el 46,7\% pertenecen al Grado de Maestro en Educación Primaria, el 27\% al Grado de Maestro en Educación Infantil, y el 26,3\% al Grado en Pedagogía. De igual forma el $73,7 \%$ está matriculado en la asignatura de "Organización del Centro Escolar" en el Grado de Infantil y Primaria; y el 26,3\% en la asignatura de "Organización y Gestión", en el Grado de Pedagogía. 


\subsection{Instrumento}

El instrumento utilizado para recoger la opinión de los estudiantes sobre el aprendizaje colaborativo es una adaptación del cuestionario elaborado por García-Valcárcel, Hernández y Recamán (2012). Dicho instrumento se compone de 27 ítems que presentan una alta fiabilidad (a de Cronbach $=0.902$ ).

El cuestionario se compone de tres partes bien diferenciadas. La primera corresponde a los datos sociodemográficos del alumnado, relacionados con la edad, el sexo, estudios previos, así como la titulación y la asignatura en la que se encuentran matriculados. La segunda parte del cuestionario contiene los 27 ítems destinados a valorar la opinión del alumnado sobre el aprendizaje colaborativo. Estos ítems siguen una escala tipo Likert con cinco opciones de respuesta, desde "totalmente en desacuerdo", hasta "totalmente de acuerdo". En la tercera parte se introducen dos preguntas abiertas para detectar los puntos fuertes y débiles del aprendizaje colaborativo desde su punto de vista.

La información se ha recogido durante el curso académico 2017-2018, gracias a la colaboración de los estudiantes implicados. El cuestionario se diseñó en la aplicación de Google Drive y se distribuyó a través de la plataforma Moodle que los estudiantes utilizan de forma habitual en las asignaturas.

\subsection{Análisis de datos}

La naturaleza del estudio y las características de la información recogida con el instrumento indicado anteriormente, nos lleva a plantear el análisis de la información bajo un enfoque cuantitativo y cualitativo. En el primero de los casos se han realizado análisis descriptivos (apoyados por la herramienta de análisis SPSS v. 21). En base a las preguntas abiertas, se ha llevado a cabo un análisis de contenido apoyado por la herramienta de análisis cualitativo Nvivo12, una herramienta adecuada para trabajar con toda la información textual. En concreto, el proceso que llevamos a cabo se resume en los siguientes análisis. Por un lado, y en lo que respecta al análisis cuantitativo, se ha solicitado la media y desviación típica de cada uno de los ítems. Por otro lado, y en lo que respecta al análisis cualitativo, el análisis del contenido de las preguntas abiertas se ha focalizado en las ventajas e inconvenientes que los alumnos perciben del aprendizaje colaborativo. Así pues, se ha extraído el contenido de estas preguntas relacionadas con estos tópicos y de manera inductiva se ha elaborado el sistema de categorías, el cual ha sido validado por expertos.

\section{Resultados}

Los resultados presentados a continuación se muestran de acuerdo al siguiente orden: a) exploración inicial de las respuestas de los estudiantes a las cuestiones cerradas del cuestionario y b) análisis de las preguntas abiertas. 


\subsection{Exploración inicial de las opiniones de los estudiantes}

Los resultados muestran una valoración positiva de esta metodología de enseñanza y aprendizaje por parte de los estudiantes. Como se recoge en la Tabla 1, las medias de la mayoría de las preguntas del cuestionario están entre 3 y 4, en una escala de 1 a 5.

En primer lugar, destacamos que un $81,7 \%$ de los estudiantes señalan estar de acuerdo o totalmente de acuerdo con la ayuda que les brinda el aprendizaje colaborativo para intercambiar diferentes puntos de vista con sus compañeros (ítem 1). Además, muestran estar de acuerdo o totalmente de acuerdo con la posibilidad de poder compartir los conocimientos adquiridos (75,9\%), y aprendizajes y responsabilidades a través de esta metodología (81,7\%). Por otro lado, el alumnado considera que el aprendizaje colaborativo le permite darse cuenta de sus errores y rectificar a tiempo, estando un 70,1\% de acuerdo y totalmente de acuerdo con esta afirmación (ítem 6). También se ha observado que cuando se les pregunta si son reticentes a trabajar en grupo, un 30,4\% manifiesta que sí, mientras que un 43\% están totalmente en desacuerdo o en desacuerdo. Este ítem presenta la media más baja $(2,75)$, y la desviación típica más elevada $(1,403)$, lo que significa que la opinión de los estudiantes está más distribuida entre las cinco opciones de respuesta. Esto mismo ocurre con el ítem 18, nos dividimos el trabajo y nos reunimos para poner en común los resultados sin más, cuya media también es más baja $(2,97)$. En este caso, el 41,6\% de los estudiantes afirma que sí, mientras que el 58,4\% no lo tiene claro o lo rechaza.

Tabla 1. Resultados del análisis descriptivo del cuestionario

\begin{tabular}{|c|c|c|c|c|c|c|c|}
\hline Ítems & Media & $\begin{array}{l}\text { Desv. } \\
\text { Tip. }\end{array}$ & $\begin{array}{c}1 \\
(\%)\end{array}$ & $\begin{array}{c}2 \\
(\%)\end{array}$ & $\begin{array}{c}3 \\
(\%)\end{array}$ & $\begin{array}{c}4 \\
(\%)\end{array}$ & $\begin{array}{c}5 \\
(\%)\end{array}$ \\
\hline $\begin{array}{l}\text { 1. El trabajo colaborativo me ayuda a } \\
\text { intercambiar los puntos de vista }\end{array}$ & 4,09 & ,865 & 1,5 & 3,6 & 13,1 & 47,4 & 34,3 \\
\hline 2. Comparto los conocimientos adquiridos & 3,90 & ,987 & 3,6 & 5,8 & 14,6 & 48,9 & 27 \\
\hline $\begin{array}{l}\text { 3. Comparto aprendizajes y } \\
\text { responsabilidades cuando trabajo en grupo }\end{array}$ & 4,09 & ,865 & 1,5 & 3,6 & 13,1 & 47,4 & 34,3 \\
\hline 4. Organizo mejor mis trabajos & 3,26 & 1,268 & 11,7 & 14,6 & 29,9 & 23,4 & 20,4 \\
\hline $\begin{array}{l}\text { 5. La calidad de los trabajos realizados } \\
\text { colaborativamente es mayor }\end{array}$ & 3,50 & 1,058 & 2,9 & 13,9 & 34,3 & 28,5 & 20,4 \\
\hline $\begin{array}{l}\text { 6. Me permite darme cuenta de mis errores } \\
\text { y rectificar }\end{array}$ & 3,87 & ,946 & 1,5 & 7,3 & 21,2 & 43,1 & 27 \\
\hline 7. Me muestro reticente a trabajar en grupo & 2,75 & 1,403 & 27,7 & 15,3 & 25,5 & 16,8 & 14,6 \\
\hline $\begin{array}{l}\text { 8. En general, prefiero trabajar } \\
\text { individualmente }\end{array}$ & 3,25 & 1,288 & 10,9 & 18,2 & 27,7 & 21,2 & 21,9 \\
\hline 9. Fomenta y motiva mi aprendizaje & 3,59 & 1,115 & 5,1 & 10,9 & 27 & 33,6 & 23,4 \\
\hline
\end{tabular}




\begin{tabular}{|c|c|c|c|c|c|c|c|}
\hline $\begin{array}{l}\text { 10. El trabajo en equipo promueve mi } \\
\text { implicación activa en el proceso de } \\
\text { aprendizaje }\end{array}$ & 3,86 & ,917 & 1,5 & 5,1 & 25,5 & 41,6 & 26,3 \\
\hline $\begin{array}{l}\text { 11. La interacción con mis compañeros } \\
\text { incrementa mi nivel de aprendizaje }\end{array}$ & 3,75 & 1,042 & 2,2 & 11,7 & 21,2 & 38,7 & 26,3 \\
\hline $\begin{array}{l}\text { 12. El trabajo en equipo me ayuda a } \\
\text { profundizar más en las ideas }\end{array}$ & 3,80 & 1,016 & 4,4 & 4,4 & 24,1 & 41,6 & 25,5 \\
\hline 13. Trabajo y aprendo más rápido & 3,41 & 1,135 & 5,1 & 14,6 & 36,5 & 21,9 & 21,9 \\
\hline $\begin{array}{l}\text { 14. Aprendo más y mejor que } \\
\text { individualmente }\end{array}$ & 3,11 & 1,258 & 12,4 & 19 & 31,4 & 19,7 & 17,5 \\
\hline $\begin{array}{l}\text { 15. Las explicaciones que se dan entre } \\
\text { los miembros del grupo facilitan mi } \\
\text { comprensión de conceptos }\end{array}$ & 3,85 & 1,028 & 2,9 & 9,5 & 15,3 & 44,5 & 27,7 \\
\hline $\begin{array}{l}\text { 16. Tengo mayor autonomía y control sobre } \\
\text { mi propio aprendizaje }\end{array}$ & 3,65 & ,990 & 4,4 & 4,4 & 32,8 & 38,7 & 19,7 \\
\hline $\begin{array}{l}\text { 17. Construyo conjuntamente el } \\
\text { conocimiento sobre el contenido a aprender }\end{array}$ & 3,69 & 1,055 & 2,9 & 10,9 & 25,5 & 35,8 & 24,8 \\
\hline $\begin{array}{l}\text { 18. Nos dividimos el trabajo y nos reunimos } \\
\text { para poner en común los resultados sin más }\end{array}$ & 2,97 & 1,345 & 19 & 20,4 & 19 & 27,7 & 13,9 \\
\hline $\begin{array}{l}\text { 19. Los trabajos colaborativos requieren } \\
\text { más dedicación por parte del alumno }\end{array}$ & 4,11 & ,888 & 2,2 & 1,5 & 16,8 & 42,3 & 37,2 \\
\hline $\begin{array}{l}\text { 20. Los alumnos que son brillantes prefieren } \\
\text { trabajar individualmente }\end{array}$ & 3,26 & 1,260 & 11,7 & 15,3 & 27,7 & 26,3 & 19 \\
\hline $\begin{array}{l}\text { 21. Los alumnos menos aventajados } \\
\text { prefieren el trabajo colaborativo para así } \\
\text { trabajar menos y aprovecharse del trabajo } \\
\text { de los compañeros }\end{array}$ & 3,72 & 1,270 & 7,3 & 10,9 & 21,2 & 23,4 & 37,2 \\
\hline $\begin{array}{l}\text { 22. Me esfuerzo en compartir conocimientos } \\
\text { cuando trabajo colaborativamente }\end{array}$ & 4,30 & ,808 & 1,5 & 1,5 & 8,8 & 42,3 & 46 \\
\hline $\begin{array}{l}\text { 23. Aprendemos a ser más tolerantes y } \\
\text { respetuosos con los demás }\end{array}$ & 3,92 & 932 & 2,9 & 4,4 & 16,8 & 49,6 & 26,3 \\
\hline $\begin{array}{l}\text { 24. Me ayuda a solucionar conflictos y } \\
\text { resolver problemas }\end{array}$ & 3,50 & 1,106 & 5,1 & 13,9 & 25,5 & 36,5 & 19 \\
\hline $\begin{array}{l}\text { 25. Aumenta mi capacidad de relación con } \\
\text { los otros miembros del grupo }\end{array}$ & 3,96 & 1,003 & 2,9 & 5,1 & 19 & 38,7 & 34,3 \\
\hline $\begin{array}{l}\text { 26. La calificación que obtengo del trabajo } \\
\text { en equipo es objetiva }\end{array}$ & 3,53 & 1,051 & 5,8 & 9,5 & 26,3 & 43,1 & 15,3 \\
\hline $\begin{array}{l}\text { 27. La calificación que obtengo del trabajo } \\
\text { en equipo es adecuada al trabajo que he } \\
\text { realizado }\end{array}$ & 3,47 & 1,176 & 8,8 & 11,7 & 21,2 & 40,1 & 18,2 \\
\hline
\end{tabular}


Si seguimos analizando los resultados (Tabla 1), observamos que un 43,10\% prefiere aprender individualmente (ítem 8), mientras que un 56,8\% no lo tiene claro o lo rechaza. Además un $57 \%$ de los estudiantes considera que la colaboración fomenta y motiva su aprendizaje (ítem 9), un 67,9\% piensa que promueve su implicación activa en el proceso de aprendizaje (ítem 10), un 65\% que incrementa su nivel de aprendizaje (ítem 11), y un $67,1 \%$ que el trabajo en equipo le ayuda más a profundizar en las ideas (ítem 11).

La moda también se establece en algunas ocasiones, en la indiferencia (3), concretamente en el ítem 13, trabajo y aprendo más rápido; y en el ítem 14, aprendo más y mejor que individualmente.

Examinando los demás ítems, se observa que más de la mitad de los estudiantes opinan que a través de la colaboración adquieren una mayor autonomía y control sobre su propio aprendizaje $(58,4 \%)$ y construyen conjuntamente el conocimiento sobre el contenido a aprender $(60,6 \%)$.

También destacamos ítems con predominio del valor máximo (5) y las medias más altas, concretamente el ítem 22, me esfuerzo en compartir conocimientos cuando trabajo colaborativamente; y el ítem 19, los trabajos colaborativos requieren más dedicación por parte del alumno. Todo ello evidencia que la colaboración requiere tiempo y esfuerzo por parte de los estudiantes.

Por otro lado, cuando se le pregunta a los estudiantes si consideran que los alumnos más brillantes prefieren trabajar individualmente, un 45,3\% afirman que sí, mientras que el $54,7 \%$ no lo tiene claro o rechaza esta idea. Sin embargo, cuando opinan sobre si los estudiantes menos aventajados prefieren la colaboración para trabajar menos, los resultados varían levemente, estando un 60,6\% de acuerdo con esta afirmación.

Además, la mayoría de los estudiantes opina que a través de la colaboración aprenden a ser más tolerantes y respetuosos $(75,9 \%)$ y que aumenta su capacidad de relación con otros miembros del grupo (73\%). A su vez, les ayuda a solucionar conflictos y resolver problemas $(55,5 \%)$.

Por último, y en relación a la calificación que obtienen en el aprendizaje colaborativo, más de la mitad de los estudiantes afirman que la calificación que obtienen es objetiva (58,4\%), y adecuada al trabajo realizado $(58,3 \%)$.

\subsection{Análisis de las respuestas de los estudiantes a las preguntas abiertas del cuestionario}

A continuación se pretende profundizar en el discurso de los estudiantes en las preguntas abiertas del cuestionario. Con toda la información, se obtiene en primer lugar, una nube de palabras con el programa Nvivo12 con el objetivo de explorar qué palabras aparecen con más frecuencia en su discurso cuando hablan de beneficios e inconvenientes del aprendizaje colaborativo. De este modo, se solicita al programa la 
obtención de una nube de 20 palabras con una longitud mínima de cuatro letras. Tal y como se muestra en la Figura 1, las palabras que aparecen con mayor frecuencia son: calidad (660 referencias), ideas (626 referencias), socialización (603 referencias), perspectivas (583 referencias), implicación (573 referencias), colaboración (569 referencias), aprendizaje (552 referencias), comunicación (549 referencias), responsabilidad (536 referencias) y motivación (534 referencias).

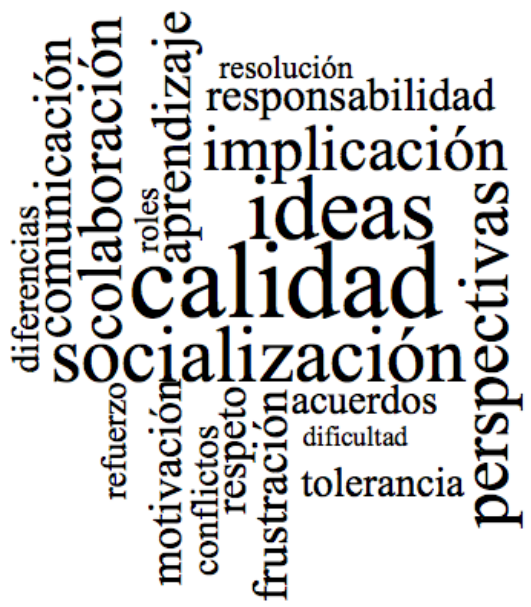

Figura 1. Nube de palabras acerca del aprendizaje colaborativo desde la perspectiva del alumnado

En segundo lugar, se obtiene con el programa Nvivo12 un mapa de categorías identificadas en el discurso de los estudiantes, organizadas en dos dimensiones principales: beneficios e inconvenientes del aprendizaje colaborativo (Figura 2).

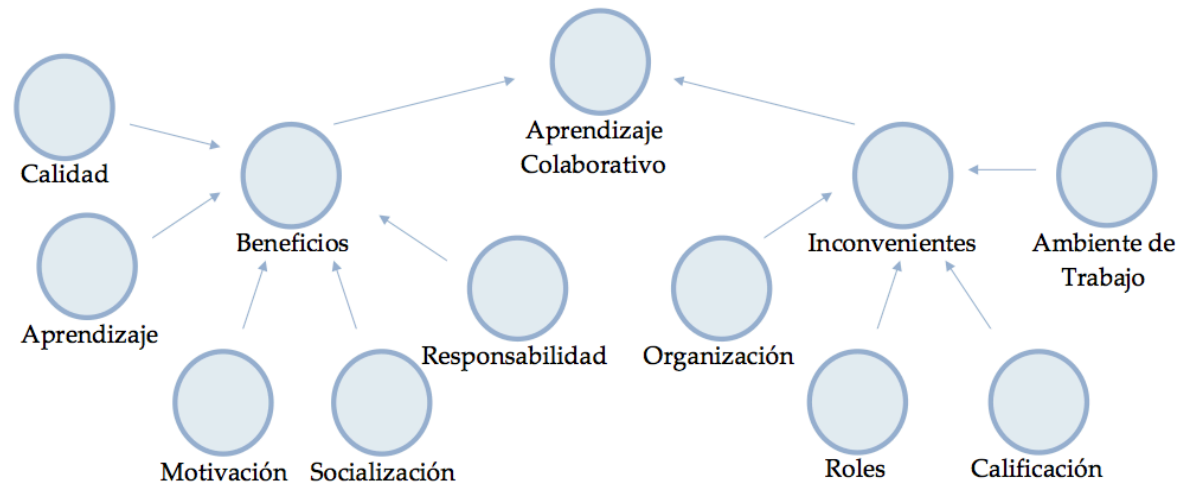

Figura 2. Mapa de categorías identificadas a partir de las preguntas abiertas del cuestionario 
A continuación se analizan cada una de estas categorías y se ejemplifican con citas textuales de las respuestas de los estudiantes para facilitar su comprensión. Los ejemplos que se aportan fundamentan las interpretaciones de quien investiga y permiten contrastar la relación dato-interpretación.

\section{Beneficios del aprendizaje colaborativo}

Los estudiantes que han participado en el estudio consideran que el aprendizaje colaborativo les ha ayudado a compartir conocimientos, intercambiar puntos de vista diferentes, obteniendo trabajos de mayor calidad:

Compartir conocimientos y opiniones diferentes, de forma que se pueden complementar para optimizar la calidad del aprendizaje colaborativo (Estudiante 57).

El hecho de realizarlo de forma colaborativa permite que el trabajo final que hemos elaborado tenga una mayor calidad gracias al intercambio de un mayor número de ideas y nuevos puntos de vista (Estudiante 8).

A su vez, los alumnos opinan que el aprendizaje que se obtiene es mayor y más enriquecedor como resultado de la interacción que se produce entre ellos, creándose vínculos duraderos. Otro aspecto que mencionan en las cuestiones abiertas es que a través de la colaboración aprenden a ayudarse los unos a los otros, y desarrollan el pensamiento crítico:

El aprendizaje es mayor. Se aprende más. Ayuda a crear vínculos y a conocer cosas que no sabías (Estudiante 9).

Aprendizaje más enriquecedor con el continuo intercambio de ideas y el aporte que cada uno de nosotros realiza al trabajo (Estudiante 73).

Los estudiantes también resaltan que el aprendizaje colaborativo es una oportunidad para mejorar las relaciones entre ellos, haciendo que se conozcan más. Del mismo modo, opinan que aprenden a respetarse y a saber escuchar:

Considero que el aprendizaje colaborativo ha mejorado la relación entre nosotros, nos hemos conocido más (Estudiante 11).

Da lugar a la aparición de valores como el respeto y el saber escuchar (Estudiante 82).

Por último, los estudiantes destacan estar más motivados cuando realizan trabajos con sus compañeros:

Mayor motivación, y, por lo general, mejores resultados que el aprendizaje individual (Estudiante 63).

La motivación ya que intentas dar todo lo que puedas de ti para que los resultados sean positivos (Estudiante 113). 


\section{Inconvenientes del aprendizaje colaborativo}

Respecto a los inconvenientes, los estudiantes señalan en algunos casos, la organización del trabajo en el aprendizaje colaborativo, y destacan especialmente algunas dificultades relacionadas con la distribución de las tareas en el grupo:

Dificultad de organización, diferencias de implicación entre los miembros, mayor pasividad de alguno de ellos, etc. (Estudiante 107).

Por otro lado, los estudiantes opinan que algunos compañeros tratan de imponer sus ideas o formas de trabajo, y no dejan que los demás colaboren:

Que haya siempre personas que no dejen colaborar a los demás libremente, exponiendo sus ideas e imponiendo las suyas (Estudiante 81).

Otro aspecto que los estudiantes resaltan tiene que ver con la calificación final del trabajo. En este sentido, demandan una calificación más individualizada y adaptada al trabajo que ha realizado cada uno en su equipo.

Unos compañeros siempre colaboran más que otros y finalmente todos obtenemos la misma nota (Estudiante 1).

La nota no es justa, porque los compañeros que no colaboran se benefician del esfuerzo de los compañeros que sí lo hacen (Estudiante 97).

Por último, los alumnos señalan que cuando trabajan con sus compañeros se producen algunos conflictos y discusiones como resultado de sus diferentes opiniones, e implicación en el trabajo.

Claramente son las discusiones que en ocasiones se producen por muchos motivos como la falta de colaboración de algunos miembros del grupo (Estudiante 110).

El aprendizaje colaborativo puede causar conflictos entre las personas que conforman el grupo (Estudiante 56).

\section{Discusión y conclusiones}

En esta investigación hemos comprobado el valor que tiene la metodología del aprendizaje colaborativo para los estudiantes, que creen fielmente en sus potencialidades, las cuales residen en el logro de objetivos más ricos en contenidos, que aseguran la calidad y exactitud de las ideas y soluciones planteadas, además de propiciar la generación de aprendizajes, donde la aportación de cada alumno es muy valiosa al no permanecer como un individuo pasivo que solo capta información. Estas conclusiones están en la línea de lo expresado por Martín y Masa (2018) en su estudio, los cuales destacan que los estudiantes aprenden más como resultado de las contribuciones de sus compañeros.

Biasutti y El-Deghaidy (2014) y Harris (2014) resaltan al respecto la importancia de la colaboración y su papel central en el aprendizaje, pues lo consideran un ingrediente clave para el éxito de los estudiantes en la sociedad global actual. Además consideran 
que la colaboración es una habilidad para la vida que reúne diversas perspectivas y conocimientos para producir mejores decisiones, y es fundamental para conseguir un trabajo más productivo y una vida social y cívica más plena (Hunter y Botchwey, 2017).

Los estudiantes también han resaltado la asunción de responsabilidades como aspecto positivo durante las actividades, y la motivación para aprender. Esta motivación e implicación se traduce en una disposición más favorable hacia las actividades y asuntos estudiados. Por otro lado, perciben esta metodología como un enfoque de trabajo que fomenta una mayor participación en las tareas académicas, además de brindarles la oportunidad de compartir e intercambiar ideas, expresar sus propias opiniones, ayudarse unos a otros, y construir de forma conjunta interactuando con otros. Todas estas habilidades son necesarias para su futuro profesional (Barba, Sonlleva y García, 2018; Gutiérrez, Román y Sánchez, 2018). Al respecto, Larmer, Mergendoller y Boss (2015) señalan que a través del aprendizaje colaborativo los estudiantes mejoran sus habilidades de cooperación y comunicación. Y es que la colaboración entre los estudiantes tiene un enorme potencial para enriquecer mutuamente las experiencias de aprendizaje de los participantes, la formación de relaciones y la interactividad (Hunter y Botchwey, 2017). Dichos factores, junto con la autonomía de los alumnos, se relacionan con un aumento en las estrategias de resolución de problemas entre los estudiantes (León, Martínez y Santos, 2018).

Thomas y MacGregor (2005) al respecto, consideran que la colaboración es una oportunidad ideal para proporcionar situaciones de solución de problemas presentes en el mundo real. Los estudiantes participan en un proceso de colaboración continua para el desarrollo de su conocimiento y comprensión, debido a sus interacciones dentro de una situación de aprendizaje que refleja el mundo que les rodea. Por ello, consideramos esencial que los docentes creen una cultura de colaboración en el aula, donde los estudiantes sientan la necesidad de interactuar con sus compañeros en las tareas que emprenden, y de ayudarse mutuamente, donde sepan que pueden cometer errores y aprender de ellos.

A su vez, el presente trabajo ha demostrado que los estudiantes encuentran varios inconvenientes durante la colaboración, tales como la intensificación de trabajo y dedicación que reclama esfuerzo y tiempo; la participación desigual en las tareas de grupo; y la falta de habilidades comunicativas y de colaboración en los estudiantes. Según Le, Janseen y Wubbels, (2017), estos son algunos de los problemas más comunes en el aprendizaje colaborativo. En este contexto, se destaca la investigación de Harris (2014), que mostró que una de las principales dificultades a la que se enfrentaron los estudiantes durante la colaboración y el trabajo en equipo, fue el hecho de que solo se implicaron los líderes de los grupos asumiendo la responsabilidad principal del trabajo. Baysura, Altun y Yucel (2016) destacan al respecto, que el motivo puede ser que algunos estudiantes no dispongan de las capacidades necesarias para contribuir por igual 
al trabajo en grupo. Krajcik y Blumenfeld (2006) por su parte, opinan que los estudiantes pueden estar acostumbrados a obtener respuestas inmediatas de los docentes y por ello, no estén tan interesados en encontrar las soluciones por sí mismos trabajando junto a sus compañeros.

Bajo nuestro punto de vista, estas dificultades pueden ser debidas, en cierto modo, a una falta de hábito y costumbre por parte del alumnado a trabajar de esta manera en el aula. Estos resultados reiteran la necesidad de la buena preparación de los estudiantes. En este contexto el rol del docente es fundamental e incluye, pues, modelar habilidades interpersonales positivas, estimular la ayuda y colaboración entre los miembros del equipo y hacer que los estudiantes practiquen dichas habilidades.

Todo ello, en definitiva, demuestra que el aprendizaje colaborativo exige una buena estructuración y seguimiento por parte del profesorado. El docente debe ser muy cuidadoso a la hora de diseñar una sesión de trabajo colaborativo, ya que son muchos los factores a tener en cuenta y que suelen pasar desapercibidos, tales como documentar los objetivos de cada actividad y criterios de evaluación que se persiguen desde el principio; planear con detalle el tiempo estimado de duración de la actividad; organizar grupos apropiados; establecer un buen clima de trabajo; y ayudar y ofrecer feedback sobre la calidad del producto elaborado por el grupo.

Por último, y en base a los resultados de este trabajo, es posible concluir que el aprendizaje colaborativo es significativo para los estudiantes cuando: a) contribuye de manera valiosa en el producto final del grupo, b) observan una relación entre su contribución y el resultado final, c) su contribución es necesaria para el producto del grupo e insustituible, y d) las exigencias de contribución no son demasiado altas.

\section{Referencias Bibliográficas}

Barba, R., Sonlleva, M. y García, N. (2018). "Presencia, participación y progreso": el aprendizaje basado en proyectos en la trayectoria de una maestra en formación. Revista Electrónica Interuniversitaria de Formación del Profesorado, 21(2), 13-25.

Baysura, O., Altun, S. y Yucel, B. (2015). Perceptions of Teacher Candidates regarding ProjectBased Learning. Eurasian Journal of Educational Research, 62, 15-36.

Biasutti, M. y El-Deghaidy, H. (2014). Interdisciplinary project-based learning: an online wiki experience in teacher education. Technology, Pedagogy and Education, 24(3), 339- 355.

Blasco, L. Buil, I., Hernández, B. y Sese, J. (2013). Using clickers in class. The role of interactivity, active collaborative learning and engagement in learning performance. Computers \& Education, 62, 102110.

De Hei, M. S., Strijbos, J. W., Sjoer, E. y Admiraal, W. (2015). Collaborative learning in higher education: Lecturers' practices and beliefs. Research Papers in Education, 30(2), 232-247.

Flick, U. (2014). La gestión de la calidad en la investigación cualitativa. Madrid. Morata. 
Fransen, J., Kirschner, P. y Erkens, G. (2011). Mediating Team Effectiveness in the Context of Collaborative Learning: The Importance of Team and Task Awareness. Computers in Human Behavior, 27(3), 1103-1113.

García-Valcárcel, A., Basilotta, V. y López, C. (2014). Las TIC en el aprendizaje colaborativo en el aula de Primaria y Secundaria. Comunicar, 21(42), 65-74.

García-Valcárcel, A., Hernández, A. y Recamán, A. (2012). La metodología del aprendizaje colaborativo a través de las TIC: una aproximación a las opiniones de profesores y alumnos. Revista Complutense de Educación, 23(1), 161-188.

Gros, B. (Ed.) (2011). Evolución y retos de la educación virtual. Construyendo el e-learning del siglo XXI. Barcelona: Editorial UOC.

Gutiérrez, I., Román, M. y Sánchez, M. (2018). Estrategias para la comunicación y el trabajo colaborativo en red de los estudiantes universitarios. Revista Comunicar, 54(26), 91-100.

Hunter, P. E. y Botchwey, N. D. (2017). Partnerships in Learning: A Collaborative Project between Higher Education Students and Elementary School Students. Innovative Higher Education, 42(1), 77-90.

Järvelä, S., Kirschner, P.A., Panadero, E. Malmberg, J. Phielix, C., Jaspers, J.,... Jarvenoja, H. (2015). Educational Technology Research Development, 63(1), 125-142.

Johnson, D. W. y Johnson, F. (2009). Joining together: Group theory and group skills. Boston: Allyn \& Bacon.

Kolloffel, B., Eysink, T. y Jong, T. (2011). Comparing the effects of representational tools in collaborative and individual inquiry learning. International Journal of Computer Supported Collaborative Learning, 6(2), 223-251.

Larmer, J., Mergendoller, J. y Boss, S. (2015). Setting the Standard for Project Based Learning: A Proven Approach to Rigorous Classroom Instruction. Novato, California: Buck Institute for Education

Le, H., Janseen, J. y Wubbels, T. (2017). Collaborative learning practices: teacher and student perceived obstacles to effective student collaboration. Cambridge Journal of Education, 48(1), 103-122.

Martín, S. y Masa, C. (2018). Collaborative learning environment (cle) en la asignatura de Fundamentos de gestión empresarial con la aplicación de m-learning y assessment feedback/ feedforward mediante pares y sistemas de autorización presencial y virtual. Perspectiva Empresarial, 5(1), 17-30. http:// dx.doi.org/10.16967/rpe.v5n1a2

McKeachie, W. J., Pintrich, P. R., Lin, Y. y Smith, D. A. (1986). Teaching and learning in the college classroom: a review of the research literature. Ann Abor, Michigan: University of Michigan, National Center for Research to improve Postsecondary Teaching and Learning.

Sabariego, M. (2004). El proceso de investigación (parte 2). En R. Bisquerra (Coord.), Metodología de la investigación educativa (pp. 127-163). Madrid: La Muralla.

Sánchez, M. C. (2015). La dicotomía cualitativo-cuantitativo: posibilidades de integración y diseños mixtos. Campo Abierto, Revista de educación, (Núm. Extra 0), 11-30.

Sobocinski, M., Malmberg, J. y Järvelä, S. (2017). Exploring temporal sequences of regulatory phases and associated interactions in low- and high-challenge collaborative learning sessions. Metacognition Learning, 12(2), 1-20. 
Yong, E. A., Cedeño, E. J., Tubay, M., y Cedeño, L. B. (2018). Trabajo colaborativo y el aprendizaje de las matemáticas en los alumnos de Economía de la UTEQ. Journal of Science and Research: Revista Ciencia e Investigación, 3(10), 10-15.

Ana $\mathrm{M}^{\mathrm{a}}$ Pinto Llorente Universidad de Salamanca,

Facultad de Educación, Departamento de Didáctica, Organización y Métodos de Investigación-Salamanca

Email: ampintoll@usal.es

ORCID :https://orcid.org/0000-0002-8538-9902

Verónica Basilotta Gómez-Pablos

Universidad a Distancia de Madrid

Facultad de Ciencias de la Salud y de la Educación,

Departamento de Educación - Madrid

Email: veronicamagdalena.basilotta@udima.es

ORCID: http://orcid.org/0000-0003-1976-4548

Vanessa Izquierdo Álvarez

Universidad de Salamanca,

Facultad de Educación, Departamento de Didáctica,

Organización y Métodos de Investigación-Salamanca

ORCID: https://orcid.org/0000-0002-0760-9017

Correspondencia

Verónica Basilotta Gómez-Pablos

Departamento de Educación

Facultad de Ciencias de la Salud y de la Educación

Vía de Servicio A-6, 15, 28400 Collado Villalba, Madrid

Data de submissão: Dezembro 2018

Data de avaliação: Março 2019

Data de publicação: Setembro 2019 